DOI: $10.5902 / 2236583464594$

\title{
Efeito de intervenções focadas nas práticas alimentares parentais sobre o comportamento alimentar e o status de peso de pré-escolares
}

\author{
Effect of parental feeding practices-focused interventions on food \\ consumption and weight status of preschoolers
}

\author{
Luísa de Freitas Ricardo Pereira, Ana Paula Carlos Cândido
}

Como citar este artigo:

PEREIRA, LUISAA F.R. ; CÂNDIDO, ANA PAULA C.; Efeito de intervenções focadas nas práticas alimentares parentais sobre o comportamento alimentar e o status de peso de pré-escolares. Revista Saúde (Sta. Maria). 2021; 47 (1).

\author{
Autor correspondente: \\ Nome: Luísa de Freitas Ricardo \\ Pereira \\ E-mail: lvisafic@gmail.com \\ Telefone: (32) 98707-7428 \\ Formação Profissional: Gra- \\ duanda em Nutrição pela \\ Universidade Federal de Juiz \\ de Fora (UFJF), Juiz de Fora, \\ Minas Gerais, Brasil. \\ Filiação Institucional: Universi- \\ dade Federal de Juiz de Fora \\ (UFJF) \\ Endereço para correspon- \\ dência: \\ Rua: São Mateus ñ: $711 / 403$ \\ - Bloco C \\ Bairro: São Mateus \\ Cidade: Juiz de Fora \\ Estado: Minas Gerais \\ CEP: 36025-00 I
}

Data de Submissão:

05/03/202 1

Data de aceite:

$21 / 06 / 2021$

Conflito de Interesse: Não há conflito de interesse

\section{(cc) BY-NC-ND}

\section{RESUMO}

Os pais desempenham papel fundamental na instalação e manutenção dos hábitos alimentares das crianças, sendo responsáveis desde a compra até a oferta dos alimentos, contexto dentro do qual estão inseridas as práticas alimentares parentais. Utilizadas com maior frequência em crianças pré-escolares, estratégias como a pressão para comer e alimentos como recompensa podem contribuir para o desenvolvimento de sobrepeso e obesidade infantil. Diante disso, têm sido desenvolvidos diferentes programas de intervenção objetivando a prevenção e/ou a redução da prevalência destas alterações, uma vez que as mesmas tem ganhado cada vez mais relevância como problemas de saúde mundiais. Nesse sentido, o presente estudo teve como objetivo avaliar o efeito de intervenções focadas nas práticas alimentares parentais sobre o comportamento alimentar e o status de peso de pré-escolares. Foi realizada uma busca, entre março e maio de 2020, nas bases de dados Scielo, PubMed e Lilacs, utilizando o termo "parental feeding practices". O total de artigos encontrados foi filtrado por ano de publicação (2016-2020), tipo de estudo, forma de acesso gratuita e faixa etária do público estudado, e posteriormente foram lidos os títulos, resumos e o texto completo dos estudos para finalizar a seleção. Ainda foram adicionados estudos de outras bases de dados, cujos anos foram anteriores ao intervalo considerado na pesquisa. A maioria das publicações encontradas mostraram redução no uso de práticas alimentares parentais inadequadas; redução no consumo de alimentos não saudáveis e aumento da ingestão de alimentos saudáveis no grupo intervenção; mas não mostraram mudanças significativas no IMC/Idade em qualquer grupo, nem diferenças significativas entre os grupos para os parâmetros referidos após os programas realizados. As intervenções focadas nas práticas alimentares parentais podem reduzir 0 uso de estratégias inadequadas pelos pais e melhorar o comportamento alimentar de crianças pré-escolares, mas não foram capazes de provocar mudanças significativas no status de peso deste público, o que mostra a necessidade de realização de mais estudos para observar efeitos neste parâmetro a longo prazo.

PALAVRAS-CHAVE: Parentalidade; Comportamento Alimentar; Peso-Estatura; Pré-Escolares.

\section{ABSTRACT}

Parents play a fundamental role in the installation and maintenance of children's eating habits, being responsible from the purchase to the offering of food, a context within which parental feeding practices are inserted. Most frequently used in preschool children, strategies such as pressure to eat and the food as reward can contribute to the development of overweight and childhood obesity. In view of this, different intervention programs have been developed aiming to prevent and/or reduce the prevalence of these changes, as they have gained increasing relevance as global health problems. In this sense, the present study aimed to evaluate the effect of interventions focused on parental eating practices on the eating behavior and weight status of preschoolers. A search was carried out, between March and May 2020, in the databases Scielo, PubMed and Lilacs, using the term "parental feeding practices". The total number of articles found was filtered by year of publication (2016-2020), a form of free access and the age group of the public studied, and later the titles, abstracts and the full text of the studies were read to finalize the selection. Studies from other databases were added, whose years were before the interval considered in the research. Most of the publications found showed a reduction in the use of inappropriate parenting eating practices; reduction in the consumption of unhealthy foods and increased intake of healthy foods in the intervention group; but they did not show significant changes in BMI / Age in any group, nor significant differences between groups for the parameters referred to after the programs performed. Interventions focused on parental feeding practices can reduce the use of inappropriate strategies by parents and improve the eating behavior of preschool children, but they have not been able to cause significant changes in the weight status of this audience, which shows the need for further studies to observe long-term effects on this parameter.

KEYWORDS: Parenting; Feeding Behavior; Weight by Height; Child, Preschool. 


\section{INTRODUÇÃO}

Os pais desempenham papel fundamental na instalação e manutenção dos hábitos alimentares das crianças, sendo responsáveis desde a compra dos alimentos que estarão disponíveis no ambiente doméstico até a oferta dos mesmos, momento no qual deve ser estimulado o consumo de alimentos saudáveis e limitada a ingestão de alimentos não saudáveis, de modo a proteger a saúde e prevenir a ocorrência de excesso de peso e de doenças.

Dentro deste contexto, estão incluídas as práticas alimentares parentais, definidas como estratégias alimentares utilizadas pelos pais durante as refeições que podem influenciar de maneira positiva ou negativa o comportamento alimentar das crianças. ${ }^{1}$ A recorrência às estratégias em questão é mais frequente na fase pré-escolar, uma vez que nesta ocorre desaceleração na velocidade de crescimento, o que condiciona a redução do apetite, além deste se tornar irregular, apresentando variações diárias ou até mesmo de uma refeição para a outra. ${ }^{2}$ Juntamente a isso, as crianças quase sempre limitam a variedade de alimentos consumidos, reduzindo a ingestão de frutas, legumes, verduras e carnes e possuindo preferência por doces e guloseimas; demoram mais tempo para realizar uma refeição e se distraem facilmente com outras atividades; e tornam-se também mais seletivas na ingestão alimentar, já sendo capazes de manifestar aceitação e recusa de alimentos, porém ainda não conseguem escolher uma alimentação balanceada e adequada ao seu crescimento e desenvolvimento. ${ }^{3}$

Entre as práticas referidas, há aquelas nas quais ocorre o diálogo entre pais e filhos e respeito aos sinais de fome e saciedade da criança, e outras que incluem pressão para comer, restrição alimentar e uso de alimentos como recompensa. ${ }^{4}$

As estratégias referidas possuem como uma das classificações marcadores de controle "diretivo" e "não diretivo". O "controle diretivo" é definido como práticas em que os pais realizam uma pressão externa e observável sobre a criança para que esta coma os alimentos ofertados, a exemplo da restrição alimentar e a pressão para comer. Já o "controle não diretivo" são as práticas pelas quais os pais incentivam a alimentação saudável através de interações motivacionais voltadas para o diálogo com a criança e a melhoria do ambiente alimentar doméstico, o que inclui permitir que as crianças escolham o que vão consumir entre alimentos com maior diversidade de nutrientes, através do aumento da disponibilidade destes; estabelecer rotinas para as refeições, se alimentando junto com as crianças e encorajar estas a consumirem alimentos saudáveis. ${ }^{5}$

As formas de controle diretivo/explícito, particularmente, podem também resultar em efeitos negativos sobre o peso das crianças quando aplicadas frequentemente, contribuindo para o desenvolvimento de excesso de peso e obesidade infantil, visto que provocam modificações no comportamento alimentar no sentido de reduzir o consumo de frutas, verduras e legumes e aumentar o consumo de alimentos ultraprocessados, principalmente doces e outras guloseimas que são utilizados como forma de chantagem. ${ }^{6}$ 
Estudo realizado no Brasil com pais de crianças de 2 a 8 anos, demonstrou que a restrição alimentar para controle de peso foi associada ao maior escore Z do IMC de pré-escolares, enquanto a orientação para alimentação saudável e a pressão para comer foram associadas ao menor escore Z do IMC, tendo em vista que esta última causa aversão aos alimentos que a criança é forçada a consumir. ${ }^{7}$

O mesmo resultado também foi encontrado em pesquisa envolvendo famílias americanas mexicanas de crianças de 8 a 10 anos que investigou a relação entre o comportamento alimentar dos pais e o status de peso das crianças, na qual a pressão para comer e o uso de alimentos para controlar o comportamento foram associados com menor escore $Z$ do IMC enquanto a restrição excessiva de alimentos foi associada com maior escore $Z$ do IMC das crianças. ${ }^{8}$

Os achados referidos convergem com os mostrados em outra publicação com díades de cuidadores e crianças de 4 a 6 anos etnicamente diversos, na qual a pressão para comer previu menores, e a restrição previu maiores, IMC e percentual de gordura corporal. ${ }^{9}$

Além destes, um trabalho desenvolvido no Reino Unido cujos participantes foram mães e seus filhos de 3 a 5 anos verificou associações semelhantes às supracitadas no que diz respeito à restrição alimentar, isto é, a maior restrição física e verbal de alimentos dos 3 aos 5 anos foi significativamente correlacionada com um escore Z de IMC posterior mais alto da criança dos 5 aos 7 anos. ${ }^{10}$

Ao mesmo tempo que os estudos em questão defendem as práticas alimentares parentais como sendo preditoras do excesso de peso e obesidade infantil, outros investigam a associação contrária ou bidirecional entre as duas questões, isto é, se o comportamento alimentar dos pais são consequência ou tanto causa quanto consequência do status de peso das crianças, porém estas pesquisas são limitadas. ${ }^{11}$

Em estudo com crianças entre 2 e 6 anos, que investigou efeitos bidirecionais entre a restrição alimentar e a altura e peso reportados pelos pais, bem como entre a pressão para comer e o peso e escore Z do IMC, indicou que percepções incorretas dos pais com relação ao status de peso da criança podem levar ao maior uso de práticas alimentares parentais com efeitos negativos sobre o peso das crianças, e que pais e filhos, influenciam-se mutuamente, uma vez que os pais apresentam comportamentos em reação ao peso das crianças, mas também influenciam o peso da criança com suas estratégias. ${ }^{12}$

Em outro estudo envolvendo com crianças aos 4 e 7 anos de idade, que visava examinar a associação bidirecional entre as práticas parentais de alimentação dos filhos e IMC aos 4 e 7 anos de idade, foram verificados resultados parcialmente similares ao anterior, com a restrição aos 7 anos de idade mostrando ser uma prática que surgiu em resposta a um IMC mais alto aos 4 anos de idade, porém a restrição aos 4 anos de idade não influenciou o IMC aos 7 anos de idade, e a pressão para comer apresentando uma associação bidirecional e negativa com o IMC, sendo esta mais forte no sentido do IMC para o uso da prática em questão. ${ }^{13}$ 
A persistência do uso de práticas inadequadas também pode fazer com que tais mudanças no status de peso, bem como em distorções a respeito dos alimentos e hábitos alimentares, perpetuem até a vida adulta. ${ }^{14}$

Diante disso, tem sido desenvolvidos diferentes programas de intervenção focados em estratégias parentais que visam a prevenção e/ou redução da prevalência de excesso de peso e obesidade infantil, uma vez que as mesmas tem ganhado cada vez mais relevância como problemas de saúde mundiais. As intervenções envolvem a realização de atividades ou consultas e a orientação, por profissionais adequados, aos pais para que estes introduzam no ambiente doméstico estratégias em que se promova o diálogo entre os pais e as crianças e o respeito aos sinais de fome e saciedade das mesmas, e reduzam os comportamentos alimentares prejudiciais, tornando a alimentação um momento prazeroso para todos.

Tendo em vista a variedade de programas existentes na atualidade, o objetivo do presente estudo é avaliar o efeito de intervenções focadas nas práticas alimentares parentais sobre o comportamento alimentar e o status de peso de pré-escolares.

\section{MÉTODO}

O presente estudo consiste em uma revisão sistemática da literatura, realizada conforme as diretrizes do Preferred Reporting Items for Systematic Reviews and Meta-Analyses (PRISMA), e que possui como etapas a definição da questão de pesquisa; a busca de evidências, que compreende a definição dos critérios de elegibilidade dos artigos e a condução de buscas nas bases de dados escolhidas, aplicando os parâmetros pré-estabelecidos e justificando as exclusões; revisão e seleção dos estudos através da leitura de títulos e resumos e/ou do texto completo; apresentação dos resultados e discussão, o que inclui preparar um resumo crítico, sintetizando as informações disponibilizadas pelos artigos incluídos na revisão; e apresentação de conclusão, informando a evidência sobre o encontrado no estudo. ${ }^{15,16,17}$

As buscas foram realizadas nas bases de dados SciELO, PubMed e LILACS no período de março a maio de 2020, utilizando o termo "parental feeding practices". Os estudos encontrados foram filtrados tendo como critérios o ano de publicação, tipo de estudo, forma de acesso ao texto completo e a idade do público estudado. Nesse sentido, foram selecionados estudos publicados entre 2016 e 2020, por serem dados mais recentes; dos tipos ensaio clínico e estudo controlado randomizado, uma vez que este último é considerado padrão-ouro entre os métodos empregados para pesquisas que avaliam a eficácia de intervenções ${ }^{18 ;}$; cujo texto completo possui acesso gratuito, entre os quais não estão incluídos os artigos disponibilizados pela Capes; e que possuem como público estudado crianças pré- escolares em decorrência do objetivo proposto.

Ao final da triagem referida anteriormente, realizou-se a leitura dos títulos, resumos e se necessário do texto completo de cada estudo restante, a partir da qual foram excluídas as duplicatas; estudos que não correspondiam 
ao objetivo proposto; estudos que estavam incompletos do ponto de vista das partes em que são divididos, isto é, introdução, metodologia, resultados, discussão e conclusão; e artigos cuja intervenção estudada envolvia apenas as crianças, sem abranger os pais.

Após esta etapa, foram incluídos estudos provenientes das bases de dados Microsoft Academic e Periódicos Capes, bem como de listas de publicações de projetos, em cuja busca também foram seguidos os procedimentos supracitados, porém considerando anos anteriores ao período de 2016 a 2020, especificamente o intervalo de 2010 a 2015, uma vez que foram encontrados poucos artigos nas três bases de dados utilizadas inicialmente no presente estudo.

\section{RESULTADOS E DISCUSSÃO}

A partir do uso do termo "parental feeding practices", foram encontrados 5568 artigos, sendo 24 provenientes da base SciELO, 5506 da PubMed e 38 da LILACS. Filtrando os estudos por data, sendo esta correspondente os anos de 2016 a 2020, foram obtidas 1835 publicações; ao limitar o tipo de estudo para ensaios clínicos e estudos controlados randomizados, 153 artigos foram identificados. Destes, 101 artigos estavam de acordo com o critério denominado textos completos e gratuitos. Ao restringir o público estudado para crianças pré-escolares, a pesquisa resultou em 45 artigos. Realizando a leitura de títulos, resumos e, se necessário, artigos completos, foram selecionadas 5 publicações. Além disso, foram identificados 2 estudos a partir da pesquisa em outras bases de dados e publicações de programas de nutrição, resultando em 7 artigos selecionados para a revisão. (Figura 1) 
Figura 1 - Fluxograma do processo de seleção dos estudos nas bases de dados

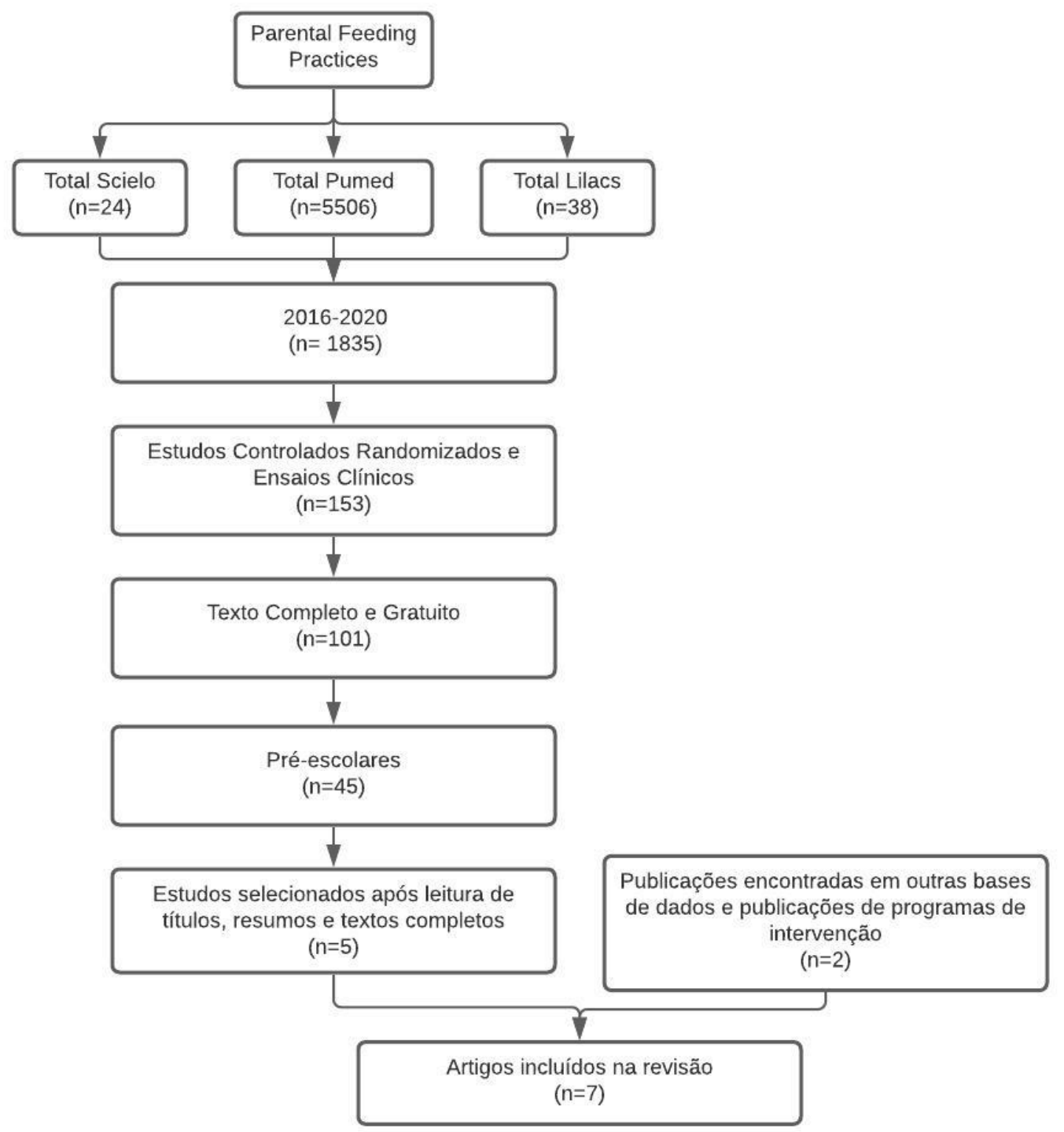

Fonte: Elaborada pelo autor

Os sete estudos selecionados foram realizados em diferentes países, sendo quatro destes nos Estados Unidos, um em Israel, um na Austrália e um no Canadá. Em relação ao público estudado, quatro publicações envolveram tanto os pais quanto seus filhos pré-escolares, duas incluíram apenas os pais de crianças nesta mesma faixa etária e uma englobou as crianças, seus pais e seus professores. Além disso, os intervalos de tempo das pesquisas foram variados, isto é, quatro delas duraram ao todo 2 a 3 anos; uma por 9 meses; uma menciona apenas a duração da intervenção, sendo esta de 16 semanas; e uma relata que os participantes foram inscritos de 2012 a 2014 e a análise dos dados começou em 2016. (Quadro 1). 
Quadro 1 - Relação das Publicações Selecionadas

\begin{tabular}{|c|c|c|c|c|}
\hline Autor (Data) & Tipo de Estudo & Local de Estudo & Tipo de Participantes & $\begin{array}{c}\text { Duração do } \\
\text { Estudo/Intervenção }\end{array}$ \\
\hline $\begin{array}{c}\text { Fisher et al. } \\
(2019)^{19}\end{array}$ & $\begin{array}{l}\text { Estudo Controlado } \\
\text { Randomizado }\end{array}$ & $\begin{array}{c}\text { Ambiente } \\
\text { universitário } \\
\text { (EUA) }\end{array}$ & $\begin{array}{l}\text { Mães Biológicas de } \\
\text { crianças de } 2 \text { a } 5 \text { anos } \\
\text { de baixa renda }\end{array}$ & $\begin{array}{l}\text { Janeiro de } 2013 \text { a } \\
\text { Setembro de } 2015 \text { (2 } \\
\text { anos e } 8 \text { meses) }\end{array}$ \\
\hline $\begin{array}{l}\text { Kaufman-Shriqui } \\
\text { et al. }(2016)^{20}\end{array}$ & $\begin{array}{l}\text { Estudo Controlado } \\
\text { Randomizado }\end{array}$ & $\begin{array}{l}\text { Escolas de baixo } \\
\text { nível sócio- } \\
\text { econômico (Israel) }\end{array}$ & $\begin{array}{c}\text { Crianças saudáveis } \\
\text { com idade entre } 4 \text { e } 7 \\
\text { anos, pais e } \\
\text { professores }\end{array}$ & $\begin{array}{l}\text { Setembro de } 2008 \text { a } \\
\text { Junho de } 2009 \text { (9 } \\
\text { meses) }\end{array}$ \\
\hline $\begin{array}{l}\text { TUCKER et al. } \\
(2019)^{21}\end{array}$ & $\begin{array}{l}\text { Estudo Controlado } \\
\text { Randomizado }\end{array}$ & $\begin{array}{c}\text { Consultórios } \\
\text { Pediátricos de } \\
\text { Atenção Primária } \\
\text { (EUA) }\end{array}$ & $\begin{array}{l}\text { Famílias com crianças } \\
\text { de } 2 \text { a } 5 \text { anos }\end{array}$ & $\begin{array}{l}\text { Julho de } 2014 \text { a Julho } \\
\text { de } 2016 \text { ( } 2 \text { anos). }\end{array}$ \\
\hline $\begin{array}{c}\text { Hammersley ML } \\
\text { et al (2019) }\end{array}$ & $\begin{array}{l}\text { Estudo Controlado } \\
\text { Randomizado }\end{array}$ & Austrália & $\begin{array}{c}\text { Pais e crianças de } 2 \text { a } \\
5 \text { anos }\end{array}$ & $\begin{array}{c}\text { Janeiro de } 2016 \text { a } \\
\text { Dezembro de } 2017\end{array}$ \\
\hline $\begin{array}{l}\text { Morshed AB RG } \\
\text { et al }(2019)^{23}\end{array}$ & $\begin{array}{l}\text { Estudo Controlado } \\
\text { Randomizado }\end{array}$ & Estados Unidos & $\begin{array}{l}\text { Crianças em idade pré- } \\
\text { escolar com risco de } \\
\text { sobrepeso e suas } \\
\text { mães com sobrepeso } \\
\text { ou obesidade } \\
\text { participantes do PAT } \\
\text { (St. Louis Parents as } \\
\text { Teachers) }\end{array}$ & $\begin{array}{c}\text { Os participantes foram } \\
\text { inscritos de } 2012 \text { a } \\
2014 \text { e a análise dos } \\
\text { dados começou em } \\
2016 .\end{array}$ \\
\hline $\begin{array}{l}\text { Østbye T et al } \\
(2012)^{24}\end{array}$ & $\begin{array}{l}\text { Estudo Controlado } \\
\text { Randomizado }\end{array}$ & $\begin{array}{c}\text { Regiões do } \\
\text { Triângulo e da } \\
\text { Tríade da Carolina } \\
\text { do Norte (EUA) }\end{array}$ & $\begin{array}{l}\text { Mulheres no pós-parto } \\
\text { e seus filhos mais } \\
\text { velhos de } 2 \text { a } 5 \text { anos. }\end{array}$ & $\begin{array}{l}\text { Novembro de } 2007 \text { a } \\
\text { Outubro de } 2009\end{array}$ \\
\hline $\begin{array}{l}\text { Walton K et al } \\
(2015)^{25}\end{array}$ & $\begin{array}{l}\text { Estudo Controlado } \\
\text { Randomizado }\end{array}$ & $\begin{array}{l}\text { Centros primeiros } \\
\text { anos do sudoeste } \\
\text { de Ontário } \\
\text { (Canadá) }\end{array}$ & $\begin{array}{l}\text { Pais canadenses de } \\
\text { crianças em idade pré- } \\
\text { escolar }\end{array}$ & $\begin{array}{c}\text { A intervenção durou } 9 \\
\text { semanas e } 0 \\
\text { acompanhamento } 9 \\
\text { meses }\end{array}$ \\
\hline
\end{tabular}

Fonte: elaborada pelo autor

Com relação ao tipo de estudo e métodos utilizados, todas as publicações consistem em estudos controlados randomizados, nos quais as populações estudadas foram divididas em grupo intervenção e grupo controle. Após a categorização, cada grupo recebeu ou foi submetido a diferentes tipos de intervenções, as quais no primeiro grupo referido consistiram em aulas, consultas, encontros e/ou orientações sobre nutrição e atividade física realizadas por profissionais capacitados. Além disso, foram avaliados antes e após as intervenções, em 3 estudos, ou antes, durante e/ ou após as intervenções, nos outros 4 estudos, parâmetros antropométricos, através da aferição de peso e estatura, além do cálculo e classificação do IMC; bem como as práticas alimentares parentais e o consumo alimentar das crianças, por meio de questionários e simulações; além de dados demográficos, atividade física, tempo de tela e horário de sono, com a finalidade de verificação dos resultados das intervenções propostas.

As crianças são multiplicadoras do conhecimento, tendo o costume de passar adiante tudo o que aprendem, o que inclui os aspectos abordados em intervenções nutricionais para promover a alimentação saudável. Porém, para 
que estas ações sejam eficazes, é necessário que os pais também sejam incentivados a aplicar mudanças nos hábitos alimentares destes e do público infantil. Por isso, se possível, é interessante que sejam feitas ações paralelas com os pais e cuidadores, abordando o mesmo assunto trabalhado com a criança, porém em uma linguagem diferente. ${ }^{26}$

A respeito da avaliação e do acompanhamento do estado nutricional de crianças, estes constituem instrumentos fundamentais para a aferição da situação de saúde da população infantil, pois permitem a identificação de situações de risco e o diagnóstico nutricional, o planejamento de ações de prevenção de doenças e promoção da saúde, e a avaliação da eficácia de programas nutricionais. ${ }^{27,28,29}$

No que se refere à avaliação do consumo alimentar de crianças e do comportamento alimentar dos pais durante as refeições, foram utilizados como recursos nos estudos a observação de refeições simuladas em laboratório e questionários respondidos pelos pais, em que se permite a avaliação apenas da ingestão alimentar ou dos dois parâmetros ao mesmo tempo. As ferramentas utilizadas nos estudos foram o recordatório de 24 horas, o Questionário de Frequência Alimentar, o Family Nutrition and Physical Activity e o Feeding Practices and Structure Questionnaire ou outros similares ao mesmo (Preschooler Feeding Questionnaire, Parental Feeding Style Questionnaire, Eating and Physical Activity Questionnaire e Questionário Online de 30 minutos). Duas publicações (22 e 24) empregaram o primeiro e um dos últimos tipos ; uma (21) utilizou o segundo e um dos últimos tipos; uma (20) recorreu apenas ao segundo e duas $(23,25)$ aplicaram apenas um dos últimos tipos. Além disso, um estudo (19) utilizou o Recordatório de 24 h e realizou observações das práticas alimentares parentais em laboratório.

A aplicação do Recordatório de 24 horas e do Questionário de Frequência Alimentar em crianças possui confiabilidade variável, uma vez que há limites de conhecimento e memória especialmente para o público em questão. Crianças menores de oito anos de idade geralmente precisam que o relato seja feito pelos pais enquanto crianças e adolescentes mais velhos são mais capazes de informar sobre a própria ingestão alimentar, o que varia conforme o nível de desenvolvimento destes indivíduos. Em decorrência disto, muitos estudos que utilizam a ferramenta supracitada para avaliar o consumo alimentar de crianças recorrem ao auxílio ou a utilização apenas dos pais, o que leva à melhoria na confiabilidade do relatório mesmo que haja possibilidade de omissão de informações por parte destes. ${ }^{30}$

No que diz respeito ao Feeding Practices and Structure Questionnaire, este tem sido considerado uma ferramenta válida e confiável para uso em mães e crianças pequenas com intuito de avaliar as práticas de alimentação dos pais, uma vez que esta resulta de 28 itens divididos em 7 subescalas, sendo estas a recompensa por comportamento, recompensa por alimentação, alimentação persuasiva, restrição aberta, restrição encoberta, configuração estruturada das refeições e tempo estruturado de refeições. ${ }^{31}$

Os resultados dos estudos com relação às práticas alimentares parentais mostraram que em cinco destes $(21,22,23,24$ e 25$)$ houve redução do uso de estratégias a exemplo do uso de alimentos como recompensa por consumir 
os alimentos ofertados ou por comportamento, uso de alimentos para acalmar a criança, restrição alimentar e pressão para comer, porém no estudo 22 não houve melhora na penúltima estratégia citada anteriormente. 0 estudo 19 mostrou aumento do uso de práticas autoritativas pelos pais, isto é, que exercem o controle sobre as crianças, mas permitem a autonomia e promovem a troca de ideias com as mesmas durante a alimentação. ${ }^{32} \mathrm{O}$ estudo 20 não teve avaliação deste parâmetro.

No que diz respeito ao consumo alimentar das crianças, quatro estudos $(20,21,22$ e 24$)$ mostraram que houve redução no consumo de alimentos não saudáveis (fast food, doces, bolos, bebidas açucaradas e alimentos prontos para o consumo) e aumento da ingestão de alimentos saudáveis (frutas e legumes) no grupo intervenção em relação ao controle, porém no estudo 24 essas diferenças não foram significativas.

Já em uma das oito publicações (19), foi verificada a redução da ingestão energética em um grupo e aumento deste mesmo parâmetro em outro grupo, isto é, a primeira modificação ocorreu no grupo intervenção e a segunda no grupo controle. Além disso, neste estudo foi considerada a ingestão energética proveniente de açúcares de adição e gorduras sólidas. Os dois estudos restantes $(23,25)$ não tiveram avaliação do parâmetro supracitado.

Em relação ao status de peso das crianças, foi demonstrado em cinco estudos (19,22,23,24 e 25) que não houve mudanças significativas no IMC (percentil/escore z) para qualquer grupo nem diferenças significativas entre os grupos para o parâmetro referido após as intervenções, seja naqueles estudos em que inicialmente a maioria das crianças se encontravam na faixa de peso saudável, seja naqueles em que inicialmente a maioria das crianças apresentavam sobrepeso ou risco de sobrepeso.

Outro estudo (21) mostrou que os parâmetros avaliados (IMC para Idade e Peso para Estatura) tiveram modificações em apenas um grupo, porém estas mudanças não foram significativas. Neste caso, o escore z do IMC reduziu no grupo intervenção e não se alterou no grupo controle. Já a publicação 20 mostrou que houve redução significativa do IMC para ambos os grupos.

Os presentes resultados foram similares aos encontrados nos estudos realizados com pré-escolares avaliados em uma revisão sistemática publicada em $2018^{33}$, correspondentes a 7 das 16 publicações incluídas nesta, no que diz respeito às práticas alimentares parentais e o status de peso das crianças, sendo este último avaliado em 3 das 7 pesquisas referidas. Ou seja, em relação ao primeiro parâmetro foram verificadas a redução significativa da pressão para comer, da restrição alimentar e do uso de alimentos para controle das emoções da criança, e quanto ao segundo parâmetro não foram encontradas mudanças significativas no IMC nem no escore Z do IMC das crianças. Em contrapartida, os resultados encontrados para o consumo alimentar das crianças divergiram do presente estudo, não mostrando melhorias significativas na ingestão de frutas e vegetais nem reduções na de alimentos ultraprocessados, o que pode estar associado ao tipo e número de aplicações da ferramenta utilizada. Ou seja, o emprego de apenas 
um recall telefônico de $24 \mathrm{~h}$, possibilita que seja avaliado o consumo alimentar de um único dia, portanto não reflete os hábitos alimentares como um todo nem as modificações deste nas crianças pré-escolares. Já no presente estudo, apesar de 2 das 7 pesquisas selecionadas não terem avaliado este parâmetro, as outras 5 restantes relatam a aplicação de pelo menos dois questionários do mesmo tipo, representando mais de um dia da semana, o que possibilita uma melhor visualização se houve modificações ou não no consumo alimentar das crianças pré-escolares.

A heterogeneidade de efeitos sobre as práticas alimentares parentais, consumo alimentar e status de peso foi associada a diferentes fatores em cada estudo verificado na presente revisão. No que diz respeito às publicações nas quais não houve modificações significativas do IMC, duas delas (16 e 19) consideram como prováveis motivos para os resultados encontrados o tamanho limitado das amostras, enquanto os outros estudos tiveram número de participantes mais significativo e que portanto não foi mostrado como provável influência nos resultados encontrados. Outro ponto destacado pelos autores foi o curto período das intervenções, o que torna difícil a ocorrência de modificações no status de peso nesse intervalo, uma vez que o acompanhamento de crianças deve ser prolongado para que sejam verificados resultados mais significativos e duradouros no que diz respeito ao peso. Também foi considerada como influência sobre os resultados encontrados a grande proporção de crianças classificadas inicialmente na faixa de peso saudável, verificada em 5 dos 7 estudos selecionados. Já o estudo 20, aborda como possível explicação o fato dos indivíduos do grupo controle, bem como os do grupo intervenção, serem previamente participantes de um programa denominado St. Louis Parents as Teachers (PAT) e continuarem sendo submetidos ao mesmo durante o período em que ocorreu o estudo. E a publicação 22 associa os resultados verificados ao fato da maioria das medidas terem sido baseadas no relato dos pais, portanto pode ter ocorrido omissão de informações por parte destes ou alterações.

Em relação ao estudo 18, no qual houve modificações, porém não significativas, do status de peso em apenas um grupo, os resultados tiveram como prováveis motivos não apenas aos fatores citados anteriormente como também a redução da ingestão de calorias pelas crianças na condição de prevenção da obesidade.

Por fim, no estudo 17 a redução significativa do peso e escore $Z$ do IMC verificada em todos os indivíduos foi associada ao fato dos dois grupos terem recebido um programa de atividade física ministrado por um profissional, o que já é obrigatório em todas as escolas de Israel, conforme é relatado na publicação referida. Além disso, deve-se também considerar o fato de que nesta não foi avaliado o efeito da intervenção sobre as práticas alimentares parentais, o que talvez poderia potencializar os resultados encontrados no grupo de intervenção e assim trazer discrepâncias significativas em relação ao grupo controle.

A presente revisão também apresentou limitações quanto à metodologia, que podem ter contribuído para as diferenças entre os resultados encontrados em cada estudo, entre as quais deve-se destacar a falta de especificação dos filtros referentes à faixa etária em duas das três principais bases de dados utilizadas, fazendo com que nestas buscas 
a maioria das publicações encontradas fossem a respeito de crianças menores de 2 anos e adolescentes, o que pode ter contribuído para a identificação, durante as buscas, de estudos com diferentes resultados para o status de peso em pré-escolares . Isto mostra a necessidade de serem realizados mais estudos envolvendo o público referido e com maior acompanhamento dos efeitos de intervenções sobre os mesmos.

\section{CONSIDERAÇÕES FINAIS}

As intervenções focadas nas práticas alimentares parentais podem reduzir o uso de estratégias inadequadas pelos pais, a exemplo da pressão para comer e do uso de alimentos como recompensa, bem como melhorar o comportamento alimentar de crianças em idade pré-escolar, aumentando o consumo de frutas e legumes e diminuindo o de alimentos ultraprocessados. Porém possuem diferentes efeitos em relação ao status de peso, não sendo capazes de promover modificações significativas neste parâmetro conforme a maioria dos estudos analisados, o que foi associado à curta duração das intervenções e a aspectos particulares de cada publicação. Nesse sentido, há a necessidade de realização de mais estudos sobre a temática supracitada, porém com intervenções duradouras, para que possa ser verificado se há efeitos significativos no status de peso de crianças pré-escolares a longo prazo.

\section{REFERÊNCIAS}

1- Pinheiro-Carozzo NP, Oliveira JHA de. Práticas alimentares parentais: a percepção de crianças acerca das estratégias educativas utilizadas no condicionamento do comportamento alimentar. Psicol Rev. 2017.

2- Vasconcelos MJOB., Barbosa JM., Pinto IDS, Lima TD, Araújo AD. Nutrição clínica: obstetrícia e pediatria. $1^{\text {a }}$ edição. Rio de janeiro (BR): Medbook; 2011.

3- Silva SMCSD, Mura JD. Tratado de Alimentação, Nutrição e Dietoterapia. $2^{a}$ edição. São Paulo (BR): Roca; 2011.

4- Loth KA, Friend S, Horning ML, Neumark-Sztainer D, Fulkerson JA. Directive and non-directive food-related parenting practices: Associations between an expanded conceptualization of food-related parenting practices and child dietary intake and weight outcomes. Appetite. 2016;

5- Murashima M, Hoerr SL, Hughes SO, Kaplowitz SA. Feeding behaviors of low-income mothers: Directive 
control relates to a lower BMI in children, and a nondirective control relates to a healthier diet in preschoolers. Am J Clin Nutr. 2012.

6- Ministério da Saúde B. Cadernos de AB: Estratégias para o cuidado da pessoa com doença crônica obesidade. Brasília: Ministério da Saúde. 2014.

7-Warkentin S, Mais LA, Latorre M do RD de O, Carnell S, Taddei JA de AC. Parents Matter: Associations of Parental BMI and Feeding Behaviors With Child BMI in Brazilian Preschool and School-Aged Children. Front Nutr. 2018.

8- Penilla C, Tschann JM, Deardorff J, Flores E, Pasch LA, Butte NF, et al. Fathers' feeding practices and children's weight status in Mexican American families. Appetite. 2017.

9-Wehrly SE, Bonilla C, Perez M, Liew J. Controlling parental feeding practices and child body composition in ethnically and economically diverse preschool children. Appetite. 2014.

10-Farrow C V., Haycraft E, Blissett JM. Observing maternal restriction of food with 3-5-year-old children: Relationships with temperament and later body mass index (BMI). Int J Environ Res Public Health. 2018.

11- Eichler J, Schmidt R, Poulain T, Hiemisch A, Kiess W, Hilbert A. Stability, continuity, and bi-directional associations of parental feeding practices and standardized child body mass index in children from 2 to 12 years of age. Nutrients. 2019.

12- Santos AF, Fernandes C, Cardia S, Fernandes M, Bost KK, Veríssimo M. Concordância entre dados antropométricos reportados vs. medidos e relação com as práticas parentais alimentares em idade pré-escolar. Análise Psicológica. 2020.

13- Afonso L, Lopes C, Severo M, Santos S, Real H, Durão C, et al. Bidirectional association between parental child-feeding practices and body mass index at 4 and 7 y of age. Am J Clin Nutr. 2016.

14- Thomé da Rosa Piasetzki C, Boff ET de O, Endruweit Battisti ID. INFLUÊNCIA DA FAMÍLIA NA FORMAÇÃO DOS HÁBITOS ALIMENTARES E ESTILOS DE VIDA NA INFÂNCIA. Rev Context Saúde. 2020. 
15- Costa AB, Zoltowski APC, Koller SH, Teixeira MAP. Construção de uma escala para avaliar a qualidade metodológica de revisões sistemáticas. Cienc e Saude Coletiva. 2015.

16-Ministério da Saúde (BR), Secretaria de Ciência, Tecnologia e Insumos Estratégicos. Departamento de Ciência e Tecnologia. Diretrizes metodológicas: elaboração de revisão sistemática e metanálise de ensaios clínicos randomizados.2012.

17- Galvão TF, Pansani TDSA, Harrad D. Principais itens para relatar Revisões sistemáticas e Meta-análises: A recomendação PRISMA. Epidemiol e Serviços Saúde. 2015; 24: 335-342

18- Sharma N, Srivastav A, Samuel A. Randomized clinical trial: gold standard of experimental designs - importance, advantages, disadvantages and prejudices. Rev. Pesqui. Fisioter. 2020;10(3).

19- Fisher JO, Serrano EL, Foster GD, Hart CN, Davey A, Bruton YP, et al. Title: Efficacy of a food parenting intervention for mothers with low income to reduce preschooler's solid fat and added sugar intakes: A randomized controlled trial. Int J Behav Nutr Phys Act. 2019.

20- Kaufman-Shriqui V, Fraser D, Friger M, Geva D, Bilenko N, Vardi H, et al. Effect of a school-based intervention on nutritional knowledge and habits of low-socioeconomic school children in Israel: A cluster-randomized controlled trial. Nutrients. 2016.

21-Tucker JM, Defrang R, Orth J, Wakefield S, Howard K. Evaluation of a primary care weight management program in children aged 2-5 years: Changes in feeding practices, health behaviors, and body mass index. Nutrients. 2019.

22-Hammersley ML, Okely AD, Batterham MJ, Jones RA. An internet-based childhood obesity prevention program (TIMe2bhealthy) for parents of preschool-aged children: Randomized controlled trial. J Med Internet Res. 2019.

23-Morshed AB, Tabak RG, Schwarz CD, Haire-Joshu D. The Impact of a Healthy Weight Intervention Embedded in a Home-Visiting Program on Children's Weight and Mothers' Feeding Practices. J Nutr Educ Behav. 2019.

24- Østbye T, Krause KM, Stroo M, Lovelady CA, Evenson KR, Peterson BL, et al. Parent-focused change to 
prevent obesity in preschoolers: Results from the KAN-DO study. Prev Med (Baltim). 2012.

25- Walton K, Jordan Filion A, Gross D, Morrongiello B, Darlington G, Simpson JR, et al. Parents and tots together: Pilot randomized controlled trial of a family-based obesity prevention intervention in Canada. Can J Public Heal. 2015.

26- Simino LAP. Educação Alimentar e Nutricional.1ª edição. Londrina (BR): Educacional;2018.

27- Gonçalves de Souza M, Ribeiro das Flores RR, de Freitas Soares PD, Ferreira Soares LJ, Avelar Monteiro P, de Jesus Teixeira Alves AM, et al. Avaliação nutricional e dietética de pré-escolares de Montes Claros, Minas Gerais. J Heal NPEPS. 2019.

28 - Rosa V da S, Sales CMM, Andrade MAC. Acompanhamento nutricional por meio da avaliação antropométrica de crianças e adolescentes em uma unidade básica de saúde. Rev Bras Pesqui em Saúde/Brazilian J Heal Res. 2017.

29 - Ministério da Saúde (PT), Instituto Nacional de Saúde Doutor Ricardo Jorge. Guia de avaliação do estado nutricional infantil e juvenil.2011.

30 -Diep CS, Hingle M, Chen TA et al. A validation study of the Automated Self-Administered 24-Hour Dietary Recall for Children (ASA24-Kids) among 9 to 11-year-old youth. J Acad Nutr Diet.2015; 115(10): 1591.

31 - Jansen E, Mallan KM, Nicholson JM, Daniels LA. The feeding practices and structure questionnaire: construction and initial validation in a sample of Australian first-time mothers and their 2-year olds. Int $\mathrm{J}$ Behav Nutr Phys Act.2014; 11(1): 72.

32 - Rochinha J, Sousa B. Os estilos e práticas parentais, a alimentação e o estado ponderal dos seus filhos. Rev. aliment. hum. 2012.

33- Matvienko-Sikar K, Toomey E, Delaney L, Harrington J, Byrne M, Kearney PM. Effects of healthcare professional delivered early feeding interventions on feeding practices and dietary intake: A systematic review. Appetite. 2018. 